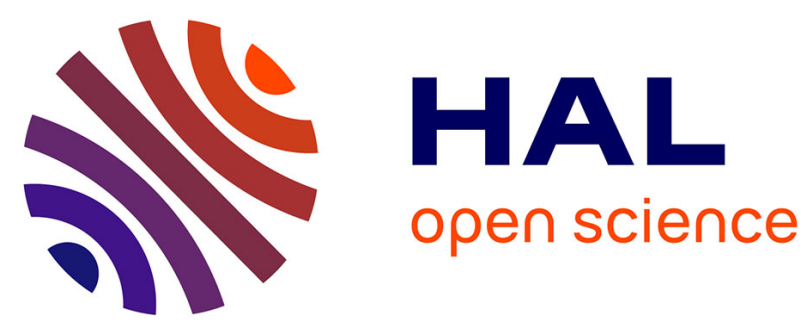

\title{
Breast cancer screening in and mutation carriers after risk reducing salpingo-oophorectomy
}

Ingrid E. Fakkert, Liesbeth Jansen, Kees Meijer, Theo Kok, Jan C. Oosterwijk, Marian J. E. Mourits, Geertruida H. Bock

\section{- To cite this version:}

Ingrid E. Fakkert, Liesbeth Jansen, Kees Meijer, Theo Kok, Jan C. Oosterwijk, et al.. Breast cancer screening in and mutation carriers after risk reducing salpingo-oophorectomy. Breast Cancer Research and Treatment, 2011, 129 (1), pp.157-164. 10.1007/s10549-011-1423-4 . hal-00618908

\section{HAL Id: hal-00618908 https://hal.science/hal-00618908}

Submitted on 4 Sep 2011

HAL is a multi-disciplinary open access archive for the deposit and dissemination of scientific research documents, whether they are published or not. The documents may come from teaching and research institutions in France or abroad, or from public or private research centers.
L'archive ouverte pluridisciplinaire HAL, est destinée au dépôt et à la diffusion de documents scientifiques de niveau recherche, publiés ou non, émanant des établissements d'enseignement et de recherche français ou étrangers, des laboratoires publics ou privés. 
Breast cancer screening in $B R C A 1$ and $B R C A 2$ mutation carriers after Risk Reducing SalpingoOophorectomy

Authors

Ingrid E Fakkert, Bsc 1,2

Liesbeth Jansen, MD, PhD 2

Kees Meijer RN, MS 2

Theo Kok, MD, PhD 3

Jan C Oosterwijk, MD, PhD 4

Marian JE Mourits, MD, PhD 5

Geertruida H de Bock, PhD 1

${ }^{I}$ Department of Epidemiology,

${ }^{2}$ Department of Surgery,

${ }^{3}$ Department of Radiology,

${ }^{4}$ Department of Clinical Genetics,

${ }^{5}$ Department of Gynaecology,

University Medical Center Groningen, University of Groningen, Groningen, the Netherlands

Address for correspondence:

Prof. Dr. G.H. de Bock, PhD

University Medical Center Groningen, University of Groningen

Department of Epidemiology

P.O. Box 30.001, 9700 RB Groningen

The Netherlands

Tel: +31503610739

Email: g.h.de.bock@epi.umcg.nl 


\section{Abstract}

Background: Breast cancer screening is offered to BRCAI and BRCA2 mutation carriers from the age of 25 years because of their increased risk of breast cancer. As ovarian cancer screening is not effective, risk-reducing salpingho-oophorectomy (RRSO) is offered after child bearing age. RRSO before menopause reduces the breast cancer risk as well as breast density. It can be questioned whether after premenopausal RRSO, the intensive breast cancer screening program needs modification.

Methods: We evaluated the effectiveness of breast cancer screening by clinical breast examination (CBE), mammography and MRI in a population of $88 B R C A 1$ and 51 BRCA2 mutation carriers who had RRSO before the age of 52. Sensitivity, specificity, positive predictive value (PPV) and negative predictive value (NPV) were calculated for each screening modality.

Results: During 422 women years, 14 breast cancers were diagnosed; 2 prevalent, 10 screen detected and 2 interval breast cancers (12 in BRCA1 and 2 in BRCA2 mutation carriers). Sensitivity, specificity, PPV and NPV for the combined screening were $85.7 \%, 97.6 \%, 30.0 \%$ and $99.8 \%$, respectively. No tumors were found with CBE, MRI had a sensitivity of $60.0 \%$ and mammography of $55.6 \%$. Off all the tumors, $60 \%$ were node positive. Discussion: Effectiveness of CBE and mammography was comparable to earlier findings. MRI screening seemed less effective than earlier findings. After RRSO, the breast cancer risk in BRCA1 and BRCA2 mutation carriers is still high enough to justify intensive breast cancer screening with MRI and mammography.

Keywords: pBSO, RRSO, BRCA1, BRCA2, breast cancer screening, MRI 


\section{Introduction}

Breast cancer is the most common cancer in women and the life time risk of developing breast cancer for individual women in western countries is approximately 12-13\% [1]. Women with a family history of breast and/or ovarian cancer can have substantially higher risks [2]. For women with a proven BRCA1 or BRCA2 mutation, life time risk can reach up to $65-80 \%$ for breast cancer and $45 \%$ for ovarian cancer by the age of 70 $[3,4]$. In the Northern Netherlands we found a cumulative breast cancer risk of $71.4 \%$ (95\% CI: $67 \%-82 \%$ ) for BRCA1 mutation carriers and 87.5\% (95\% CI: 82\%-93\%) for BRCA2 mutation carriers by age of 70 [5]. For ovarian cancer these risks were 58.9\% (95\% CI: 54\%-64\%) and 34.5\% (95\% CI: 25\%-44\%) respectively [5]. Female mutation carriers are counselled on different risk reducing strategies, e.g. screening or prophylactic surgery. Prophylactic mastectomy strongly reduces the breast cancer risk, with about $90 \%$ at the age of 70 when conducted at a mean age of 38 [6,7]. Intensive breast cancer screening in mutation carriers consists of annual MRI, mammography and clinical breast examination (CBE) and can reach a sensitivity of more than $90 \%$ in finding early stage breast cancer in mutation carriers [8-12]. For ovarian cancer it was recently shown that current screening protocols are ineffective in detecting early stage ovarian cancer in mutation carriers [13, 14] and the only effective strategy to prevent ovarian cancer death is a risk reducing salpingo-oophorectomy (RRSO), which is eventually chosen by approximately $70 \%$ of these women $[15,16]$.

When this procedure is performed before the age of 50 years, the risk of breast cancer is also reduced, with up to $50 \%$, depending on the timing of RRSO [17]. Nevertheless, Breast cancer screening before the age of 50 is indicated for women with a breast cancer risk that is 3 times higher than the population risk [18,19] BRCAl and $B R C A 2$ mutation carriers continue to receive the same three modality breast screening program after RRSO as they had before.

Intensive breast cancer screening combining MRI, mammography and CBE was proven to be effective in women at high risk for breast cancer [8-11]. One of the reasons that MRI was introduced in the screening program is that mammography is less effective in younger premenopausal women with higher breast density, as is the case in BRCA1/2 mutation carriers. We hypothesize that the breast cancer screening protocol might need adaptation in women after RRSO, because of the lower breast cancer incidence and the lower breast density after surgical menopause. The aim of this study is therefore to determine the effectiveness of breast cancer screening after RRSO in BRCA1/2 mutation carriers.

\section{Materials and methods}

\section{Study population}

To determine the effectiveness of breast cancer screening in BRCA1/2 mutation carriers after RRSO, a retrospective cohort study was performed in BRCA1 or BRCA2 mutation carriers who underwent a RRSO. All women who were younger than 52 years of age at RRSO (which is the mean age at menopause the Netherlands) were included. Results from subsequent breast cancer screening visits at the family cancer clinic from the University Medical Centre Groningen between January 1995 and the end of September 2009 were analyzed. Women needed to have at least 1 breast eligible for screening. Women with uni- or bilateral breast conserving therapy were included. The use of hormonal replacement therapy (HRT) was recorded and this was not an 
exclusion criterion. Women in whom ovarian cancer was diagnosed during RRSO were not included in the study.

\section{Surveillance protocol}

In our family cancer clinic, RRSO was initially an option from the age of 35-40 years in BRCA1 mutation carriers and from the age of 40-45 years in BRCA2 mutation carriers. As of 2007, counseling has moved towards a specific advice to have RRSO around the age of 40, because ovarian screening appeared not effective in reducing ovarian cancer death $[13,14]$. However, the actual timing of this decision depends on many personal circumstances such as previous breast cancer, marital status, previous or planned pregnancies and mental acceptance of this definitive procedure. The operative procedure is a daycare surgical procedure, performed by laparoscopy [20].

As of 1995, annual clinical breast examination (CBE) by oncologic surgeons and nurse practitioners was performed together with annual mammography from the age of 25. From 1999, annual MRI was added to the protocol in a part of our population that participated in the MRISC study [8,12], After 2005 it is advised to screen $B R C A 1 / 2$ mutation carriers with annual CBE and MRI from age 25 till 60, to which mammography is added from age 30 onwards [18,19]. At our centre, MRI and mammography imaging is ideally performed alternating, i.e. with a six months interval.

\section{Variables and endpoints}

Information on date of birth, mutation status and personal oncologic history, date of RRSO and HRT use were collected for all women. For previous breast cancers, we collected information about age at diagnosis and therapy. We reviewed every visit were CBE, mammography and/or MRI was performed. For all these visits, the reason for visiting (screening or interval visit due to signs or symptoms), the performed diagnostic procedures and the outcomes of these investigations were collected. The result of a CBE consult is considered possibly malignant in case of referral for further investigation. The results of mammography and MRI screening are scored in a standardized way, according to the Breast Imaging Reporting and Data System (BI-RADS) classification. BI-RADS categories vary between 0 to $\mathrm{V}$ and BI-RADS category III or higher are considered abnormal and indicate further analysis by ultrasound, MRI and/or tissue sampling [21-23].

When additional diagnostic procedures were performed, the type of procedure and the findings were noted. Findings could be 'no malignancy' (when no malignancy was found at the additional diagnostic procedures), 'suspect for malignancy' (when more additional investigation was necessary) and 'malignancy' (as confirmed by biopsy). For each new breast cancer, tumour size in millimeters and the presence or absence of lymph node metastases in the (sentinel) axillary lymph nodes was noted.

\section{Data collection}

All relevant data were retrieved from the patients' hospital file. Physician's letters, pathology reports and imaging reports were used to collect detailed information about above mentioned variables and endpoints as defined below. Data from all consecutive screening visits after RRSO were entered into an SPSS database. Protection of the patient's identity was guaranteed by a patient identification number. Those numbers were only retraceable to an individual woman when entered in the hospital database, which is only accessible for medical 
staff from the hospital, having a personal account. Complying with Dutch law, no further Institutional Review Board approval was needed.

\section{Statistical analysis}

Duration of follow-up was calculated for each woman. Follow-up time was calculated from the date of RRSO to the following endpoints: date of prophylactic mastectomy, date of ablation or date of last screening visit. Newly detected malignancies were defined as prevalent, screen-detected or interval cancers. Women were assigned to 2 groups, based on their mutation (BRCA1 or BRCA2). Characteristics of women are presented with median and range when they are continuous variables. For the other variables numbers are given and percentage for each mutation group. Differences between groups were calculated with the Kruskall Wallis test for continuous variables and $\chi^{2}$ tests for the percentages within mutation groups. To compare the 3 different screening modalities, we calculated the sensitivity, specificity, PPV and NPV of each screening modality. For these 4 outcomes, 95\% confidence interval $(95 \% \mathrm{CI})$ was calculated with the Wilson score interval method. For MRI and mammography, calculations were made for BI-RADS III and IV as cut off point for a positive (suspect) test. Statistic analyses were performed using the software of SPSS 16.0 for Windows and the VassarStats website [24].

\section{Results}

\section{Population characteristics}

Of all the mutation carriers who were screened at the UMCG after RRSO during the study period, 139 women met our inclusion criteria; 88 (63.3\%) BRCA1 mutation carriers and 51 (36.7\%) BRCA2 mutation carriers (Table 1). BRCA1 mutation carriers had a median age at RRSO of 40.5 years (range 30-51 years) and BRCA2 mutation carriers had a median age of 42 years (range 33-51 years) Of all women, $18 \%$ had a history of breast cancer, BRCA1 mutation carriers more often than BRCA2 mutation carriers ( $22.7 \%$ and $9.8 \%$ respectively, $\mathrm{p}=0.081)$. Of all women, BRCA1 mutation carriers reported significantly more often breast cancer before and at the age of 45 years than BRCA2 mutation carriers. ( $80.0 \%$ and $40.0 \%$ respectively, $\mathrm{p}=0.034)$.

\section{Screening visits and events during follow-up}

The total follow-up in this study was 5064 months (422 women years). The median follow-up was 24.0 months per woman (range 2-236 months). Women were censored because of preventive mastectomy ( $\mathrm{n}=32,23.0 \%)$, breast cancer $(n=2,1.4 \%)$, referral to another hospital $(n=7,5.0 \%)$ or loss to follow-up $(n=10,7.2 \%)$, the other women were still in screening after the end of our study (Table 2). In the women for whom screening ended after detection of breast cancer, one underwent mastectomy and one did not undergo further screening after being diagnosed with metastases after breast cancer in history.

\section{Malignancies after RRSO}

In a total of 1,146 hospital visits, 1,191 screening modalities (CBE, MRI or mammography) were performed in 422 women years. During the total screening period, 14 new breast cancers were detected: 12 new tumors and two prevalent tumors, both within 6 months after RRSO. The median time between RRSO and detection of a 
new tumor was 16.5 months. Three patients with a new breast cancer had a history of breast cancer before RRSO. All of the three were contra lateral new breast cancers, there were no local recurrences. In 6 of the 10 invasive cancers the lymph nodes were positive. One of the 2 interval cancers was node positive. Of the 14 women who were diagnosed with breast cancer after RRSO, 5 used HRT. One of these breast cancers was an interval tumor.

\section{Performance of the screening methods}

The performance of the 3 screening modalities is summarized in table 3 . The overall sensitivity of the breast cancer screening program is $85.7 \%$. Two tumors were interval cancers No tumors were found by CBE (Table 3). This gives CBE a sensitivity of $0 \%$, a specificity of $97.7 \%$, a PPV of $0 \%$ and a NPV of 98.1 . In 9 women with breast cancer after RRSO, mammography screening was conducted and 5 of the 9 cancers were identified by mammography. This gives mammography a sensitivity of $55.6 \%$, a specificity of $98.4 \%$, a PPV of $50.0 \%$ and a NPV of 98.8. In 10 women with breast cancer after RRSO MRI screening was conducted, 6 of these cancers were identified by MRI. This gives MRI a sensitivity of $60.0 \%$, a specificity of $95.9 \%$, a PPV of $35.3 \%$ and a NPV of $98.5 \%$.

Of the 2 interval tumors, the first, (20 mm, node positive), was detected 5 months after screening CBE.

Mammography screening had not yet been performed. This was in 1999 and MRI was not yet part of the screening protocol at that time. Five years after diagnosis, this patient died of metastatic disease after a relapse in the mastectomy scar. The second interval cancer $(11 \mathrm{~mm}$, node negative) was detected by the patient herself 2 weeks after a negative CBE screening. MRI had not been conducted and the last mammography was 17 months before the new tumor was found.

\section{Screening visits and additional investigation}

Of the 138 women who attended screening after RRSO, 127 women had at least one screening CBE, 117 women had at least one screening mammography and 109 women had at least one screening MRI (Table 4). The total number of screening investigations was highest for CBE (583), and lowest for MRI (278). CBE generated the least additional diagnostic procedures $3 \%$ of the screening visits led to additional research. For MRI $21 \%$ of the screening visits led to additional research and for mammography, $14 \%$ of the screening visits led to additional research. In the study period, a total of 27 invasive diagnostic procedures (15 fine needle aspirations and 12 biopsies) were conducted, also most after MRI. Most additional diagnostic procedures were performed by using ultrasound (64 times).

While no new cancers where found with CBE, CBE was carried out as additional investigation 11 times. Of the tumors found during the screening period, 5 were palpable when CBE was carried out after suspect findings at mammography or MRI. In 3 of these cases, the women had had a negative CBE screening in the month before the suspect findings.

When screening consisted of MRI and mammography, 43 screening visits were needed to find 1 new breast cancer. When CBE was added to the screening, 85 screening visits were needed to find 1 new breast cancer.

\section{Discussion}


In this group of 139 BRCA1/2 mutation carriers who had had RRSO at a premenopausal age and were under surveillance at our Family Cancer Clinic, 14 breast cancers were detected in 14 women during 442 women years. There were 2 prevalent, 10 screen detected and 2 interval breast cancers. The program sensitivity was $85.7 \%$ and the specificity $97.5 \%$. For the individual screening modalities, the sensitivity for CBE was $0 \%$ (95\% CI: $0 \%$ $32.1 \%$ ), for mammography $55.6 \%$ (95\% CI: $22.7 \%-79.9 \%$ ), and for MRI $60.0 \%$ (95\% CI: $27.4 \%-86.3 \%$ ). Of all these cancers, $70 \%$ was high grade (III) and $60 \%$ of the tumors were node positive.

The program sensitivity of screening with MRI, mammography and CBE was 85.7\% (95\% CI: 56.2\% - 97.6\%). Earlier studies on screening effectiveness in women at high risk of breast cancer found sensitivities varying from $91.1 \%$ to $97.7 \%$ [8-11], which seems to be higher. Only one study on the long-term effectiveness of the MRISC study found a total program sensitivity of $86.6 \%$ [12]. The study population in these previous studies was more diverse, consisting of pre- and postmenopausal women. Also, their studies contained mutation carriers, but also women at high risk for breast cancer without mutation. In our study, all women were postmenopausal after premenopausal RRSO and they were all mutation carriers. These factors may have contributed to the differences in effectiveness.

Both Kriege et al. and Rijnberger et al. present results of the MRISC study. Of the 2275 participants in these studies, 148 were from our family cancer clinic.(12) Kriege et al. noted that $19 \%$ of the mutation carriers had RRSO in history [8]. The amount of women analyzed in both the MRISC study and our study is probably small. A main factor that might have contributed to the lower sensitivity of our surveillance program is that we evaluated the program sensitivity of the screening over a period of 14 years (1995 - 2009) during which screening guidelines changed several times. MRI was offered from 1999 to a subset of women as part of the MRISC study, and as of 2005 it is offered to all women at high risk. One interval cancer was found in 1999. Excluding this tumor from the analysis, would increase the program sensitivity of our screening from $84.6 \%$ to 91.7\%, which is in the range that can be expected. Another consideration is that the four series mentioned [8-11] are prospective studies with a strict screening protocol in which every woman receives MRI, mammography and $\mathrm{CBE}$ at regular intervals. In daily practice, many factors may influence the regularity of the screening visits. Planning MRI, mammography and CBE on the first visit is sometimes difficult because of agenda and capacity issues, women do not always attend appointments, or there may be contra indications for MRI scanning. It is reasonable to assume that the second interval cancer is related to "protocol deviances". This interval cancer was detected by the patient herself, 2 weeks after a negative CBE screening, and her last mammography was 17 months before the new tumor was found. Though this woman was in screening from 2005 to 2007, she was not screened by MRI.

For the separate screening modalities, the sensitivity of $0 \%$ (95\% CI: $0 \%-32.1 \%$ ) for CBE found in our study was comparable to sensitivities of 9.1-17.8\% found in other studies [8,9]. In general, CBE is considered to have no additional benefit over mammography, whereas it does increase the false positive rate [25,26]. When CBE was excluded from program sensitivity analyses, sensitivity did not change. This would mean exclusion of 538 CBE screenings visits in which no new tumors were detected. Three of the new tumors found by imaging were palpable at diagnosis (CBE was conducted as additional research), while CBE screening within the month before diagnosis was negative. Also, $\mathrm{CBE}$ has proven to be useful in detecting locoregional recurrences and contralateral breast cancer in an early stage in women with breast cancer in history (eg. after mastectomy, breast 
conserving therapy and radiotherapy) [27]. This indicates that CBE should not be eliminated from our postRRSO screening protocol, especially in women with previous breast cancer.

The sensitivity of 55.6\% (95\% CI: $22.7 \%$ - 79.9\%) for mammography was not significantly different from sensitivities of 32\%-40\% reported in previous studies.(8-11). Long-term follow-up in the MRISC study revealed a sensitivity for mammography of $25 \%$ in BRCA1 mutation carriers.(12) This is much lower than the sensitivity found in our study, while the majority of tumours we found, were also in BRCA1 mutation carriers. Due to the lower breast density after RRSO, we expected that the sensitivity of mammography in our population would be higher than in other high risk screening studies. The sensitivity of mammography we found was indeed higher than previously reported though the difference was not significant. This may be due to small numbers, or to the fact that about $47 \%$ of the women used HRT after RRSO, which is known to preserve breast density in postmenopausal women [28].

The sensitivity of $60.0 \%$ (95\% CI: $27 \%$ - 86\%) for MRI in our study was lower (though not significant) than the sensitivities for MRI reported in previous studies, ranging from 71.1\%-77.0\% [8-10] and significantly lower than the sensitivity of $90.7 \%$ for MRI found by Kuhl et al [11]. It is reasonable to suppose that the effectiveness of MRI screening in daily practice is less than the performance of MRI screening in prospective trials. At our centre, MRI software, protocols and scanners evolved in the past years. It is likely that MRI sensitivity will improve in the future along with experience and further improvement of MRI technology. Another issue is that, 2 of the 4 tumors that where false negative on MRI were full DCIS. Most tumors missed on MRI in the MRISC study were also DCIS, which indicates that MRI is significantly less reliable in detecting DCIS [26]. This is illustrated by the fact that for invasive tumors only, MRI sensitivity in our study is $75 \%$, which lowers to $60.0 \%$ (95\% CI: $27.4 \%$ - 86.3\%) when DCIS are included. Rijnsburger et al. found an MRI sensitivity of $66.7 \%$ for BRCA1 mutation carriers and $69.2 \%$ for BRCA2 mutation carriers, which was significantly higher than the sensitivity of $25 \%$ they found for mammography. In addition, they showed that MRI enabled the detection of breast tumors in a more favorable stage than mammography. They suggest that MRI-screening should be considered biannually in certain categories [12].

In our study, tumor size was also comparable to other studies, with $83.3 \%$ of the tumors after RRSO being smaller than $20 \mathrm{~mm}$, compared to percentages ranging from $75 \%-94 \%$ in other studies [8-11]. However, $60 \%$ of the tumors in our study were node positive, while others found percentages of 15.4-36.0\%, [8-12]. These findings need further evaluation.

This is the first study on the effectiveness of breast cancer screening with CBE, mammography and MRI, in BRCA1/2 mutation carriers who had undergone RRSO at premenopausal age, conducted in daily practice and not in the context of a clinical trial.

It is an interesting finding that MRI sensitivity in this study was slightly lower than MRI sensitivity in earlier trials. The question is if this difference is caused by protocol deviances in general practices our by effects generated by RRSO.

Also, mammography sensitivity seems to be higher after RRSO than before. This might be caused by lower breast density after RRSO, but this should be evaluated during long term follow-up.

We used the number of new tumors detected by screening to evaluate the effectiveness of the surveillance program. Eventually, the purpose of screening is to increase survival after breast cancer. For evaluating the effect 
of survival, long term follow-up is needed. We continue to collect data of this study population in a prospective database.

To conclude, the sensitivity of three-modality breast cancer screening in BRCA1/2 mutation carriers after RRSO is lower than breast cancer screening before RRSO. It is the lower sensitivity of MRI that contributed largely to the lower sensitivity of the total screening program, while mammography sensitivity increases after RRSO. In our post RRSO population, CBE did not contribute to tumor detection. Our findings suggest that intensive breast cancer screening with both MRI and mammography should be continued in BRCA1/2 mutation carriers, after RRSO. 
Table 1: Characteristics of the women at baseline $(\mathrm{N}=139)$

\begin{tabular}{|c|c|c|c|c|}
\hline & $B R C A 1(\mathrm{~N}=88)$ & $B R C A 2(\mathrm{~N}=51)$ & Total $(\mathrm{N}=139)$ & Statistics \\
\hline $\begin{array}{l}\text { Age at RRSO in } \\
\text { years } \\
\text { Median(Range) }\end{array}$ & $40.5(30-51)$ & $42.0(33-51)$ & $41.0(30-51)$ & $\mathrm{P}=0.059$ \\
\hline $\begin{array}{l}\text { Previous breast } \\
\text { cancer } \\
\text { No } \\
\text { Yes unilateral } \\
\leq 45 \text { years } \\
\text { Yes bilateral } \\
\leq 45 \text { years }\end{array}$ & $\begin{array}{c}77.3 \%(68 / 88) \\
15.9 \%(14 / 88) \\
85.7 \%(12 / 14) \\
6.8 \%(6 / 88) \\
100 \%(6 / 6) \\
\end{array}$ & $\begin{array}{l}90.2 \%(46 / 51) \\
9.8 \%(5 / 51) \\
40.0 \%(2 / 5) \\
- \\
-\end{array}$ & $\begin{array}{c}82.0 \%(114 / 139) \\
13.7 \%(19 / 139) \\
73.7 \%(14 / 19) \\
4.3 \%(6 / 139) \\
100 \%(6 / 6) \\
\end{array}$ & $\begin{array}{l}\mathrm{P}=0.081 \\
\mathrm{P}=0.034 *\end{array}$ \\
\hline $\begin{array}{l}\text { Age at diagnosis } \\
\text { first breast cancer } \\
\text { in years }(\mathrm{N}=25) \\
\text { Median } \\
\text { (Range) }\end{array}$ & $40.0(30-49)$ & $46.0(34-50)$ & $41.0(30-50)$ & $P=0.143$ \\
\hline $\begin{array}{l}\text { Breast cancer } \\
\text { therapy** } \\
\text { BCT unilateral } \\
\text { Mast unilateral } \\
\text { BCT bilateral } \\
\text { BCT and Mast }\end{array}$ & $\begin{array}{l}40.0 \%(8 / 20) \\
40.0 \%(8 / 20) \\
15.0 \%(3 / 20) \\
5.0 \%(1 / 20)\end{array}$ & $\begin{array}{l}80.0 \%(4 / 5) \\
20.0 \%(1 / 5) \\
- \\
-\end{array}$ & $\begin{array}{l}48.0 \%(12 / 25) \\
36.0 \%(9 / 25) \\
12.0 \%(3 / 25) \\
4.0 \%(1 / 25)\end{array}$ & $P=0.427$ \\
\hline $\begin{array}{l}\text { HRT use }^{\#} \\
\text { No } \\
\text { Yes }\end{array}$ & $\begin{array}{l}51.2 \%(41 / 80) \\
48.8 \%(39 / 80)\end{array}$ & $\begin{array}{l}56.2 \%(27 / 48) \\
43.8 \%(21 / 48)\end{array}$ & $\begin{array}{l}53.1 \%(68 / 128) \\
46.9 \%(60 / 128)\end{array}$ & $\mathrm{P}=0.583$ \\
\hline
\end{tabular}

* $\mathrm{P}<0.05$ is significant. Mann-Whitney $\mathrm{U}$ test for continuous variables, Pearsons' $\chi^{2}$ test for proportions.

** BCT: Breast Conserving Therapy, Mast: Mastectomy, if noted as BCT and Mast, 1 breast is treated with BCT and 1 with Mast.

\# Hormonal replacement therapy after RRSO. 
Table 2: Follow-up

\begin{tabular}{|l|l|l|l|}
\hline & BRCA1 (N=88) & BRCA2 (N=51) & Total (N=139) \\
\hline $\begin{array}{l}\text { Follow-up in months } \\
\text { Total } \\
\text { Median (range) }\end{array}$ & $\begin{array}{l}3621 \\
29.0(2-236)\end{array}$ & $\begin{array}{l}1443 \\
20.0(2-143)\end{array}$ & $\begin{array}{l}5064 \\
24.0(2-236)\end{array}$ \\
\hline $\begin{array}{l}\text { Prophylactic } \\
\text { mastectomy }\end{array}$ & $20.5 \%(18 / 88)$ & $27.5 \%(14 / 51)$ & $23.0 \%(32 / 139)$ \\
\hline $\begin{array}{l}\text { Screening ended after } \\
\text { breast cancer }\end{array}$ & $2.3 \%(2 / 88)$ & - & $1.4 \%(2 / 139)$ \\
\hline $\begin{array}{l}\text { Referral to other } \\
\text { hospital }\end{array}$ & $6.8 \%(6 / 88)$ & $2.0 \%(1 / 51)$ & $5.0 \%(7 / 139)$ \\
\hline $\begin{array}{l}\text { Lost to follow-up } \\
\text { Still in screening }\end{array}$ & $9.1 \%(8 / 88)$ & $3.9 \%(2 / 51)$ & $7.2 \%(10 / 139)$ \\
\hline
\end{tabular}


Table 3: Performance of the screening tests

\begin{tabular}{|c|c|c|c|}
\hline & Cancer present & Cancer absent & Total \\
\hline \multicolumn{4}{|c|}{$\begin{array}{l}\text { CBE } \\
11 \text { cancers ( } 2 \text { interval), } 9 \text { invasive, } 2 \text { DCIS }\end{array}$} \\
\hline Positive & - & 13 & 13 \\
\hline Negative & 11 & 559 & 570 \\
\hline Total & 11 & 572 & 583 \\
\hline \multicolumn{2}{|c|}{ Sensitivity: $0 \%$ (95\% CI: $0 \%$ - 32\%) } & \multicolumn{2}{|c|}{ PPV: $0 \%(95 \%$ CI: $0 \%-28 \%)$} \\
\hline \multicolumn{4}{|c|}{$\begin{array}{l}\text { Mammography BI-RADS } \geq \text { III } \\
9 \text { cancers (no interval), } 7 \text { invasive, } 2 \text { DCIS }\end{array}$} \\
\hline Positive & 5 & 5 & 10 \\
\hline Negative & 4 & 316 & 320 \\
\hline Total & 9 & 321 & 330 \\
\hline \multicolumn{2}{|c|}{$\begin{array}{l}\text { Sensitivity: } 55.6 \% \text { (95\% CI: } 23 \%-85 \%) \\
\text { Specificity: } 98.4 \% \text { (95\% CI: } 96 \%-99 \% \text { ) }\end{array}$} & \multicolumn{2}{|c|}{$\begin{array}{l}\text { PPV: } 50.0 \% \text { (95\% CI: } 20 \%-80 \%) \\
\text { NPV: } 98.8 \% \text { (95\% CI: } 97 \%-100 \%)\end{array}$} \\
\hline \multicolumn{4}{|c|}{$\begin{array}{l}\text { MRI BI-RADS } \geq \mathrm{III} \\
10 \text { cancers (no interval), } 7 \text { invasive, } 3 \text { DCIS, }\end{array}$} \\
\hline Positive & 6 & 11 & 17 \\
\hline Negative & 4 & 257 & 261 \\
\hline Total & 10 & 267 & 278 \\
\hline \multicolumn{2}{|c|}{$\begin{array}{l}\text { Sensitivity: } 60.0 \% \text { (95\% CI: } 27 \%-86 \% \text { ) } \\
\text { Specificity: } 95.9 \% \text { (95\% CI: } 93 \%-98 \%)\end{array}$} & \multicolumn{2}{|c|}{$\begin{array}{l}\text { PPV: } 35.3 \%(95 \% \text { CI: } 15 \%-61 \%) \\
\text { NPV: } 98.5 \% \text { (95\% CI: } 96 \%-100 \%)\end{array}$} \\
\hline \multicolumn{4}{|c|}{$\begin{array}{l}\text { Total screening } \\
14 \text { cancers ( } 2 \text { interval), } 11 \text { invasive, } 3 \text { DCIS }\end{array}$} \\
\hline Positive & 12 & 28 & 40 \\
\hline Negative & 2 & 1149 & 1151 \\
\hline Total & 14 & 1177 & 1191 \\
\hline \multicolumn{2}{|c|}{$\begin{array}{l}\text { Sensitivity: } 85.7 \% \text { (95\% CI: 56\% - 98\%) } \\
\text { Specificity: } 97.6 \% \text { (95\% CI: } 97 \% \text { - 98\%) }\end{array}$} & \multicolumn{2}{|c|}{$\begin{array}{l}\text { PPV: } 30.0 \%(95 \% \text { CI: } 17 \%-47 \%) \\
\text { NPV: } 99.8 \%(95 \% \text { CI: } 99 \%-100 \%)\end{array}$} \\
\hline
\end{tabular}


Table 4: Screening visits and additional research

\begin{tabular}{|l|l|l|l|l|l|l|l|l|}
\hline $\begin{array}{l}\text { Screening } \\
\text { modality }\end{array}$ & Visits & CBE & $\begin{array}{l}\text { Mammo } \\
\text { graphy }\end{array}$ & MRI & $\begin{array}{l}\text { Ultra } \\
\text { sound }\end{array}$ & FNA* & Biopsy & Total \\
\hline CBE (N=127) & 583 & 4 & 2 & - & 7 & 2 & 2 & 17 \\
\hline $\begin{array}{l}\text { Mammography } \\
(\mathrm{N}=117)\end{array}$ & 330 & 2 & 3 & 2 & 31 & 3 & 4 & 45 \\
\hline MRI (N=109) & 278 & 5 & 4 & 5 & 27 & 10 & 6 & 57 \\
\hline Total & 1191 & 11 & 9 & 7 & 64 & 15 & 12 & 118 \\
\hline
\end{tabular}

*FNA: Fine Needle Aspiration 


\section{References:}

1. Nederlandse kankerregistratie (2008), Borstkankerincidentie in vrouwen per 100 000. IKCnet. http://www.ikcnet.nl/ Accesed 17 februari 2011

2. Claus EB, Risch N, Thompson WD (1994) Autosomal dominant inheritance of early-onset breast cancer. Implications for risk prediction. Cancer 73:643-51.

3. Antoniou A, Pharoah PD, Narod S, Risch HA, Eyfjord JE, Hopper JL, Loman N et al (2003) Average risks of breast and ovarian cancer associated with BRCA1 or BRCA2 mutations detected in case Series unselected for family history: a combined analysis of 22 studies. Am J Hum Genet 72:1117-30.

4. Chen S, Parmigiani G (2007) Meta-analysis of BRCA1 and BRCA2 penetrance. J Clin Oncol 25:1329-33.

5. Van der Kolk DM, De Bock GH, Leegte BK, Schaapveld M, Mourits MJ, De Vries J, Van der Hout AH, Oosterwijk JC (2010) Penetrance of breast cancer, ovarian cancer and contralateral breast cancer in BRCA1 and BRCA2 families: high cancer incidence at older age. Breast Cancer Res Treat 124:643-51

6. Rebbeck TR, Friebel T, Lynch HT, Neuhausen SL, van 't Veer L, Garber JE, Evans GR et al (2004) Bilateral prophylactic mastectomy reduces breast cancer risk in BRCA1 and BRCA2 mutation carriers: the PROSE Study Group. J Clin Oncol 22:1055-62.

7. Heemskerk-Gerritsen BA, Brekelmans CT, Menke-Pluymers MB, van Geel AN, Tilanus-Linthorst MM, Bartels CC, Tan M et al (2007) Prophylactic mastectomy in BRCA1/2 mutation carriers and women at risk of hereditary breast cancer: long-term experiences at the Rotterdam Family Cancer Clinic. Ann Surg Oncol 14: 3335-44.

8. Kriege M, Brekelmans CT, Boetes C, Besnard PE, Zonderland HM, Obdeijn IM, Manoliu RA et al (2004) Efficacy of MRI and mammography for breast-cancer screening in women with a familial or genetic predisposition. N Engl J Med 351: 427-37.

9. Warner E, Plewes DB, Hill KA, Causer PA, Zubovits JT, Jong RA, Cutrara MR et al (2004) Surveillance of BRCA1 and BRCA2 mutation carriers with magnetic resonance imaging, ultrasound, mammography, and clinical breast examination. JAMA 292: 1317-25.

10. Leach MO, Boggis CR, Dixon AK, Easton DF, Eeles RA, Evans DG, Gilbert FJ et al (2005) Screening with magnetic resonance imaging and mammography of a UK population at high familial risk of breast cancer: a prospective multicentre cohort study (MARIBS). Lancet 365:1769-78.

11. Kuhl CK, Schrading S, Leutner CC, Morakkabati-Spitz N, Wardelmann E, Fimmers R, Kuhn W et al (2005) Mammography, breast ultrasound, and magnetic resonance imaging for surveillance of women at high familial risk for breast cancer. J Clin Oncol 23:8469-76.

12. Rijnsburger AJ, Obdeyn I, Kaas R, Tilanus-Linthors MMA, Boetes C, Loo CE et al (2010) BRCA1Associated breast cancers present differently from BRCA2-associated and familial cases: long-term follow-up of the Dutch MRISC study. J Clin Oncol 28:5265-73

13. Van de Velde NM, Mourits MJ, Arts HJ, de VJ, Leegte BK, Dijkhuis G, Oosterwijk JC et al (2009) Time to stop ovarian cancer screening in BRCA1/2 mutation carriers? Int J Cancer 124:919-23.

14. Hermsen BB, Olivier RI, Verheijen RH, van Beurden M, de Hullu JA, Massuger LF, Burger CW, Brekelmans CT, Mourits MJ, de Bock GH, Gaarenstroom KN, van Boven HH, Mooij TM, Rookus MA (2007) No efficacy of annual gynaecological screening in BRCA1/2 mutation carriers; an observational follow-up study. Br J Cancer 96:1335-42.

15. Meijers-Heijboer H, Brekelmans CT, Menke-Pluymers M, Seynaeve C, Baalbergen A, Burger C, Crepin E et al (2003) Use of genetic testing and prophylactic mastectomy and oophorectomy in women with breast or ovarian cancer from families with a BRCA1 or BRCA2 mutation. J Clin Oncol 21: 1675-81. 
16. Metcalfe KA, Birenbaum-Carmeli D, Lubinski J, Gronwald J, Lynch H, Moller P, Ghadirian P et al (2008) International variation in rates of uptake of preventive options in BRCA1 and BRCA2 mutation carriers. Int J Cancer 122: 2017-22.

17. Rebbeck TR, Kauff ND, Domchek SM (2009) Meta-analysis of risk reduction estimates associated with riskreducing salpingo-oophorectomy in BRCA1 or BRCA2 mutation carriers. J Natl Cancer Inst 101: 80-7.

18. Kwaliteitsinstituut voor de Gezondheidszorg CBO (2008) Richtlijn mammacarcinoom 2008. CBO. http://www.cbo.nl/Downloads/328/rl_mamma_08.pdf Accessed 17 February 2011

19. National Institute of Clinical Excellence (NICE) (2006) National Institute of Clinical Excellence (NICE) guideline CG41 Familial Breast Cancer 2004 updated in 2006. NICE. http://www.nice.org.uk/CG41 Accessed 17 February 2011

20. Kenkhuis MJ, de Bock GH, Elferink PO, Arts HJ, Oosterwijk JC, Jansen L, Mourits MJ (2010) Short-term surgical outcome and safety of risk reducing salpingo-oophorectomy in BRCA1/2 mutation carriers. Maturitas 66: 310-4.

21. Tardivon AA, Athanasiou A, Thibault F, El Khoury C (2007) Breast imaging and reporting data system (BIRADS): magnetic resonance imaging. Eur J Radiol 61:212-5.

22. Liberman L, Menell JH (2002) Breast imaging reporting and data system (BI-RADS). Radiol Clin North Am 40:409-30.

23. Obenauer S, Hermann KP, Grabbe E (2005) Applications and literature review of the BI-RADS classification. Eur Radiol 15:1027-36.

24. Lowry R. Clinical calculator 1: from an observed sample - estimates of population prevalence, sensitivity, specificity, predictive values, and likelihood ratios. VassarStats. http://faculty.vassar.edu/lowry/clin1.html Accessed 17 February 2011.

25. Chiarelli AM, Majpruz V, Brown P, Thériault M, Shumak R, Mai V (2009) The contribution of clinical breast examination to the accuracy of breast screening. J Natl Cancer Inst 101:1236-43.

26. Obdeijn IM, Loo CE, Rijnsburger AJ, Wasser MNJM, Bergers E, Kok T et al (2010) Assessment of falsenegative cases of breast MR imaging in women with a familial or genetic predisposition. Breast Cancer Res Treat 119:399-407

27. Lu W, de Bock GH, Schaapveld M, Baas PC, Wiggers T, Jansen L (2010) The value of routine physical examination in the follow up of women with a history of early breast cancer. Eur J Cancer (in press)

28. Sendağ F, Coşan Terek M, Ozşener S, Oztekin K, Bilgin O, Bilgen I et al (2001) Mammographic density changes during different postmenopausal hormone replacement therapies. Fertil. Steril. 76;445-50. 